\title{
Validation of water vapour profiles from GPS radio occultations in the Arctic
}

\author{
M. Gerding and A. Weisheimer \\ Alfred Wegener Institute for Polar and Marine Research, Research Division \\ Potsdam, Potsdam, Germany
}

\begin{abstract}
The relevance of water vapour in atmospheric physics and climate research contrasts strongly with the availability of humidity data in the Arctic. The most extensive humidity data set is based on approx. 80 radiosonde stations north of $60^{\circ} \mathrm{N}$, but suffers from two major problems: Sensor diversity and sensor limitations under Arctic conditions, on the one hand, and lacking radiosonde launch sites in the Arctic Ocean and the Greenland iceshield, on the other hand. Calibration free satellite borne instruments like the GPS receiver onboard CHAMP prevent from both handicaps. Comparisons between radiosonde data and GPS-based humidity profiles are presented for single occultations as well as averaged data from the proofof-concept experiment GPS/MET and the recent CHAMP satellite mission. The effects of low absolute humidity and uncertain meteorological analyses are examined using additional information from a regional climate model. For the observations of CHAMP in summer 2001, a general dry bias has been found if compared with radiosonde data, apparent both in single and mean profile intercomparisons. In contrast, during February 1997 GPS/MET data show slightly higher humidity in the mid-troposphere, if compared with model data and objective analyses.

(Keywords: Water vapour, Arctic, Validation, Regional Model, Radiosondes)
\end{abstract}

\section{Introduction and data coverage}

Water vapour is the most important greenhouse gas in the Earth's atmosphere, inducing about two third of the natural greenhouse effect. For anthropogenic greenhouse effect, General Circulation Models predict the strongest signal in the Arctic region (Walsh 2001). Therefore, the monitoring of the Arctic water vapour distribution is required to seperate anthropogenic effects from natural variability and to understand the radiative feed back from increasing water vapour content due to rising temperatures, waning sea ice extend etc. 


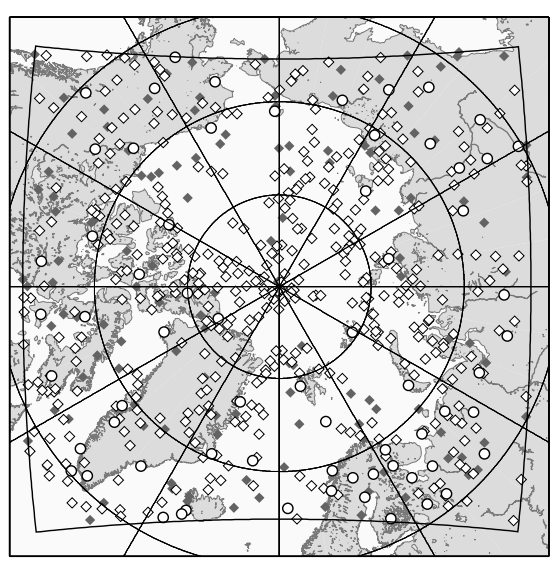

Fig. 1. Distribution of GPS/MET radio occultations (filled diamonds), CHAMP radio occultations (open diamonds), and radiosonde stations (circles). The model domain is marked by the large quadrangle. Periods of the GPS RO data are given in the text.

GPS radio occultation (RO) soundings provide profiles of atmospheric refractivity with global coverage. Calibration-free measurements and retrievals recommend this technique especially for climate applications (Yuan et al. 1993). In this paper, the reliability and limitations of specific humidity retrieval from RO combined with analyses from European Center for Medium Range Weather Forecast (ECMWF) will be investigated for Arctic conditions. RO humidity data has been provided by GeoForschungsZentrum Potsdam. Figure 1 shows the distribution of 101 individual GPS RO from the GPS/MET prime-time IV period between February 2-16, 1997 and 470 CHAMP RO between May 14-June 10 and August 26-28, 2001. Only occultations within the domain of the regional climate model HIRHAM have been taken into account (see quadrangle in Fig. 1).

66 radiosonde launch sites are situated in the examined area (Fig. 1), most of them performing one to four ascends per day. The meteorological sondes from the Global Telecommunications System (GTS) still provide the most comprehensive humidity data set in the Arctic, although they are of limited reliability (Elliot and Gaffen 1991; Miloshevich et al. 2001). The data set for February 1997 was extended by sondes launched during the "Match campaign" (Schulz et al. 2000) and the FASTEX campaign (http://www.cnrm.meteo.fr/dbfastex/).

Objective analyses from the ECMWF model have been evaluated for February 1997. Additional information is provided by the high resolution climate model HIRHAM (Christensen et al. 1996; Dethloff et al. 1996, 2001).

\section{CHAMP observations in summer 2001}

61 out of 470 CHAMP radio occultations in the Arctic have been performed simultaneously with radiosonde launches within a spatiotemporal radius of $+/-3 \mathrm{~h}$ 

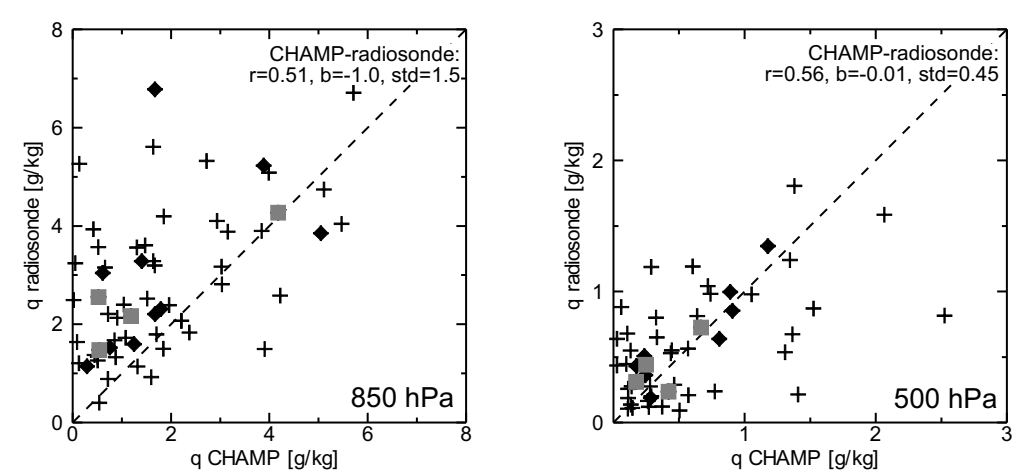

Fig. 3. Specific humidity from radiosonde and CHAMP radio occultations in the Arctic. All radiosondes launched within $3 \mathrm{~h}$ and $300 \mathrm{~km}$ distance from the occultation point are used. Closer 'matches' are marked (1h, 100km: grey squares; $2 \mathrm{~h}, 200 \mathrm{~km}$ : diamonds). r: correlation coefficient, b: bias, std: standard deviation

and $300 \mathrm{~km}, 16$ profiles within $+/-2 \mathrm{~h} / 200 \mathrm{~km}$, and only 4 profiles within $+/-1 \mathrm{~h} /$ $100 \mathrm{~km}$. Fig. 2 compares the specific humidities in 850 and $500 \mathrm{hPa}$ altitude. The correlation between the data sets is moderate (correlation coefficient of 0.51/0.56 in $850 / 500 \mathrm{hPa}$ ). In the lower troposphere CHAMP data has been found about $1 \mathrm{~g} / \mathrm{kg}$ drier than radiosondes, but about equal in the mid-troposphere. In both altitudes the standard deviation between the data sets is high $(1.5 / 0.45 \mathrm{~g} / \mathrm{kg}$ in $850 / 500 \mathrm{hPa}$ ). The deviation decreases with decreasing distances of the sounded air parcels. Individual profiles are not compared here because of remaining spatiotemporal differences. They are of less importance for climatological studies.

Fig. 3 shows mean humidity profiles from all radiosondes and CHAMP radio occultations for the area in Fig. 1, separated by land and coastal/marine regions. Compared with Fig. 2, the number of used single profiles is largely increased, providing the best available data set for climatological investigations in the Arctic region. It is obvious that the CHAMP data is drier by up to $50 \%$ compared with radiosonde humidity. Part of this difference may result from the lack of radiosonde data from the dry, central Arctic Ocean and the Greenland iceshield.
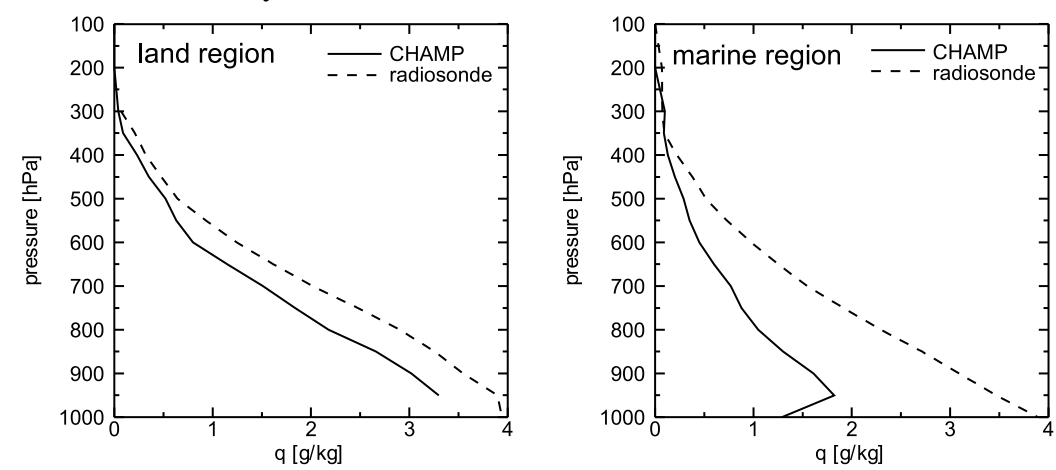

Fig. 2. Mean specific humidity profile for specific Arctic regions for May/June 2001. The data has been analysed on 17 pressure levels $(1000-100 \mathrm{hPa})$ 


\section{GPS/MET observations in February 1997}

Wintertime GPS RO are available from GPS/MET for February 1997. Comparisons of single profiles with radiosondes suffer from the low number of RO. Therefore, objective analyses from ECMWF have been used for comparison, along with results from the regional model HIRHAM. Specific humidity from RO is compared with model data for the low and mid troposphere (Fig. 4). The model results have been spatiotemporal interpolated to the location of radio occultation. The correlation between the data sets is rather poor in the lower troposphere $(0.36 / 0.35$ for comparison with ECMWF/HIRHAM), but higher in $500 \mathrm{hPa}(0.71 / 0.68)$. In this altitude the GPS/MET data gives slightly higher humidity. For the lower troposphere there are some indications for a small dry-bias of the GPS/MET data, but this difference is not significant because of the high standard deviation.

Comparisons of regional mean profiles (not shown here) reveal that the differences between models and GPS RO occur especially above the Arctic land regions, while above open sea-water all data sets agree well. Radiosonde data available above the Arctic land area confirm the GPS/MET observed profiles and the differences from meteorological analyses. This indicates that in the data sparse Arctic region the analysis results are dominated by the underlying atmospheric model and the assimilation of observational data might have a limited effect only.
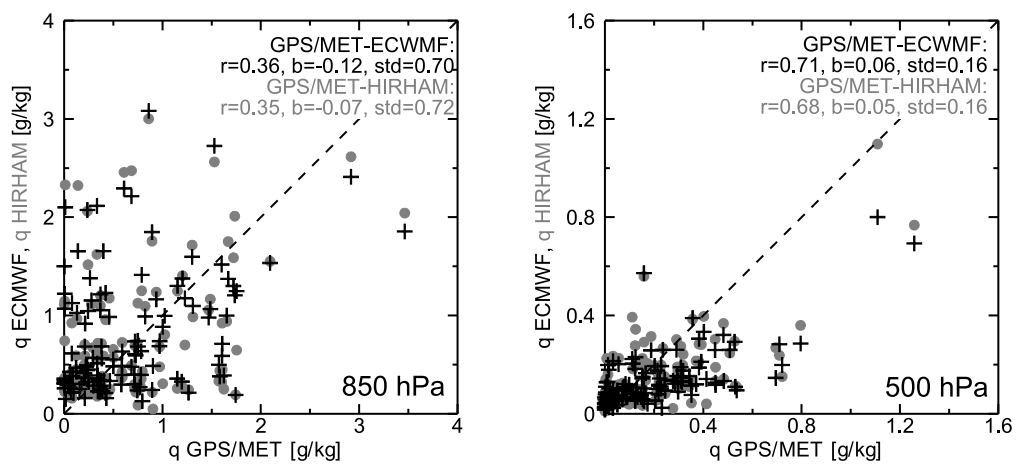

Fig. 4: Specific humidity from ECMWF analyses, HIRHAM, and GPS/MET RO in the Arctic. r: correlation coefficient, b: bias, std: standard deviation

\section{Discussion and conclusions}

Comparison between GPS RO and radiosonde data suffer in general from the limited number of joint soundings and accurate matches of the sounded air parcels. The high standard deviations and only moderate correlation coefficients displayed in Fig. 2 may result from high spatiotemporal variability in the humidity distribution. But still, a significant dry bias of the CHAMP data remains. On the other hand, standard meteorological radiosondes are known to be of limited reliability especially in cold and dry environments (Elliot and Gaffen 1991). However, radi- 
osondes themselves tend to produce dry-biased results if the sounded air gets colder (Miloshevich et al. 2001). Therefore a wet-bias of the radiosondes seems to be unlikely. Negative specific humidities are a well-known problem of GPS RO soundings with several possible reasons, like multipath effects, non-spherical symmetry of the Earth's atmosphere, or uncertainties in the ancillary temperature fields used in the retrieval (see, e.g. Rocken et al. 1997; Marquardt et al. 2000). We have neglected negative values for averaging, but cannot exclude that also other profiles are affected by the above mentioned problems.

The humidity inversion found in the marine humidity profile indicates a stable boundary layer above the ice covered part of the ocean. Typically, this area is only poorly masked by radiosonde data, which therefore does not exhibit any inversion. On the other hand, we cannot exclude that these might be erroneous data due to reflections and multipath effects of the GPS signal (Beyerle and Hocke 2001). Further analysis e.g. with atmospheric models is needed to answer this question.

Comparisons between meteorological analyses and GPS/MET RO show large differences in individual profiles, like between CHAMP RO and radiosonde data. The rather good correlation between GPS/MET \& ECMWF data and between GPS/MET \& HIRHAM data in the middle troposphere reveals the high potential of RO for climate applications. The weaker correlation in the 850-hPa-layer could be explained with the above mentioned reflections and a vanishing GPS signal near the ground (Rocken et al. 1997). Achieving a higher antenna gain, the CHAMP instrument overcame this problem. In contrast to the global findings of Kursinski and Hajj (2001), the Arctic humidity estimates from GPS/MET exhibit slightly wetter conditions than analysed by ECMWF. This coincides with the colder tropospheric air modeled by ECMWF and HIRHAM.

This study suggests that ECMWF objective analyses are of limited accuracy in the data sparse Arctic region and additional observations are needed. Also the humidity retrieval of GPS RO suffers from uncertainties in the analyses. Therefore, new approaches have been discussed in literature using statistical methods or ground-based meteorological data instead (Healy and Eyre 2000; O'Sullivan et al. 2000). Further investigation will show whether these methods can overcome the problems in the remote Arctic region.

The limitations of objective analyses or ancillary observational data will mostly affect the single RO profile data. However, averaged, climatological humidity data from longer periods are still valuable and one of the most promising applications for the GPS radio occultation technique.

\section{References}

Beyerle G, Hocke K (2001) Observation and simulation of direct and reflected GPS signals in radio occultation experiments. Geophys Res Lett 28: 1895-1898

Christensen JH, Christensen OB, Lopez P, van Meijgaard E, Botzet M (1996) The HIRHAM4 regional atmospheric climate model. In: DMI Sci Rep 96-4, Dan Meteorol Inst, Copenhagen 
Dethloff K, Rinke A, Lehmann R, Christensen JH, Botzet M, Machenhauer B (1996) Regional climate model of the Arctic atmosphere. J Geophys Res 101: 23,401-23,422

Dethloff K, Abegg C, Rinke A, Hebestadt I, Romanov VF (2001) Sensitivity of Arctic climate simulations to different boundary-layer parametrizations in a regional climate model. Tellus 53A: 1-26

Elliot WP, Gaffen DJ (1991) On the utility of radiosonde humidity archives for climate studies. B Am Meteorol Soc 72: 1507-1520

Healy SB, Eyre JR (2000) Retrieving temperature, water vapour and surface pressure information from refractive-index profiles derived by radio occultations: A simulation study. Q J R Meteorol Soc 126: 1661-1683

Kursinski ER, Hajj GA (2001) A comparison of water vapor derived from GPS occultations and global weather analyses. J Geophys Res 106: 1113-1138

Marquardt C, Labitzke K, Reigber C, Schmidt T, Wickert J (2001) An assessment of the quality of GPS/MET radio limb soundings during February 1997. Phys Chem Earth (A) 26: 125-130

Miloshevich LM, Vömel H, Paukkunen A, Heymsfield AJ, Oltmanns SJ (2001) Characterization and correction of relative humidity measurements from Vaisala RS80-A radiosondes at cold temperatures. J Atmos Ocean Tech 18: 135-156

O'Sullivan DB, Herman BM, Feng D, Flittner DE, Ward DM (2000) Retrieval of water vapor profiles from GPS/MET radio occultations. Bull Amer Meteorol Soc 81: 10311040

Rocken C, Anthes R, Exner M, Hunt D, Sokolovskiy S, Ware R, Gorbunov M, Schreiner W, Feng D, Herman B, Kuo YH, Zou X (1997) Analysis and validation of GPS/MET data in the neutral atmosphere. J Geophys Res 102: 29,849-29,866

Schulz A et al. (2000) Match observations in the Arctic winter 1996/97: High stratospheric ozone loss rates correlate with low temperatures deep inside the polar vortex. Geophys Res Lett 27: 205-208

Walsh JE (2001) Simulation of the Arctic in global climate models. In: Second Wadati Conference on Global Change and the Polar Climate. Tsukuba Science City, Japan

Yuan L, Anthes RA, Ware RH, Rocken C, Bonner WD, Bevis MG, Businger S (1993) Sensing climate change using the Global Positioning System. J Geophys Res 98: $14,925-14,937$

\section{Acknowledgement}

We would like to thank H. Deckelmann (AWI, Potsdam) for computational support. Radiosonde data has been kindly provided by the British Atmospheric Data Centre, Meteo France, the Danish Meteorological Institute, the Norwegian Institute for Air Research, and the National Institute for Environmental Studies (Japan). We are grateful to GeoForschungsZentrum Potsdam for supplying the radio occultation profiles of specific humidity. The HIRLAM/HIRHAM system was developed by the HIRLAM project group, a cooperative project of the national weather services in Denmark, Finland, Iceland, Ireland, The Netherlands, Norway, Sweden, and Spain. This work was carried out within the HGF "GPS Atmospheric Sounding Project" (BMBF grant No. 01SF9922/2). 